\title{
Differential Relationships among Facets of Alexithymia and BDNF- and Dopamine-Related Polymorphisms
}

\author{
Nancy S. Koven, Leah H. Carr \\ Program in Neuroscience, Bates College, Lewiston, USA. \\ Email: nkoven@bates.edu \\ Received December $5^{\text {th }}, 2011$; revised January $29^{\text {th }}, 2012$; accepted February $16^{\text {th }}, 2012$
}

\begin{abstract}
Alexithymia refers to a cluster of emotion-related deficits such as difficulty attending to and identifying one's feelings. Although not a diagnosable psychiatric condition, alexithymia is considered a personality risk factor for multiple pathologies, including somatoform, substance use, eating, and mood disorders. Evidence suggests heritability, but few studies have examined the influence of specific genes on alexithymic traits. Candidate genes explored thus far include those involved in modulation of brain-derived neurotrophic factor (BDNF) and dopamine, two neurotransmitters whose functions have been implicated in human emotion processing. This study investigated the relationship between the C270T polymorphism of the BDNF gene, facets of alexithymia, and possible interactions with the COMT, DAT1, and ANKK1 genes in a sample of 130 healthy adults. Given the multidimensionality of the alexithymia construct and its overlap with the related constructs of emotional intelligence and mood awareness, we used principal components analysis to derive Clarity of Emotion and Attention to Emotion as specific facets of alexithymia. Results showed that the C270T C/C genotype group had lower Clarity of Emotion scores relative to the $\mathrm{C} / \mathrm{T}$ genotype group, even after covarying for COMT, DAT1, and ANKK1 genotypes. Dopamine-related genes had no association with alexithymia dimensions, nor did they interact with the C270T polymorphism to predict Clarity of Emotion. Although the molecular mechanisms by which this polymorphism influences BDNF are unknown, this study suggests a role for BDNF in modulating aspects of alexithymia. We discuss these results in the context of BDNF's trophic effects in the nervous system.
\end{abstract}

Keywords: Brain-Derived Neurotrophic Factor; Alexithymia; Dopamine; Polymorphism

\section{Introduction}

As a trait composed of emotion-related deficits involving difficulty attending to and identifying one's feelings, alexithymia has been associated with problematic coping patterns across a range of medical and psychiatric illnesses, including chronic pain, breast cancer, somatization disorders, substance use disorders, eating disorders, and mood disorders [1]. Alexithymia can arise from brain trauma, sexual assault, and childhood exposure to an emotionally dysfunctional family [2-4]. However, with estimates of alexithymia at $10 \%$ of the general population [5], alexithymia also manifests in individuals without any of the above risk factors, necessitating other etiological explanations. While results of twin studies suggest genetic factors [6,7], very few studies to date have examined the influence of specific genes on alexithymic traits.

Candidate genes explored thus far include those involved in modulation of brain-derived neurotrophic factor (BDNF) and dopamine (DA), two neurotransmitters whose functions have been implicated in human emotion processing [8]. BDNF has received considerable atten- tion in the neurogenetic literature for its trophic effects on dopaminergic, cholinergic, and serotonergic neurons. Several polymorphisms of the BDNF gene, located on chromosome $11 \mathrm{p} 14.1$, have been identified: a dinucleotide repeat $(\mathrm{GT})_{\mathrm{n}}$ polymorphism, a Val66Met $(196 \mathrm{G} / \mathrm{A})$ polymorphism, $-374 \mathrm{~A} / \mathrm{T}$ and $-256 \mathrm{G} / \mathrm{A}$ polymorphisms, and a $-270 \mathrm{C} / \mathrm{T}$ substitution. Of these, only the Val66Met polymorphism has been examined in the context of alexithymia, with the finding that presence of at least one 66Met allele, which functionally leads to reduced BDNF secretion, is associated with alexithymia and, in particular, difficulty identifying one's feelings [9]. With respect to DA, analysis of the chromosome 22q11.21-q11.23 gene coding for catechol-O-methyltransferase (COMT), an enzyme responsible for synaptic catecholamine degradation particularly in the prefrontal cortex (PFC), has led to mixed results. One study found that the Val allele of the COMT Val108/158Met polymorphism, which results in faster DA catabolism and therefore less DA availability in the PFC, is associated with alexithymia [10]. A similar study, however, found no association [11]. Finally, 
the A1 allele of ANKK1 gene located on chromosome 11q22-q23, which has been linked to reduced receptor binding potential of the $\mathrm{D}_{2}$ receptor [12], is associated with alexithymia [9].

The interaction of BDNF 66Met and ANKK1 A1 genotypes as predictors of alexithymia has inspired a multistep theoretical model postulating that reduced BDNF levels lead to reduced dopaminergic activity in the $\mathrm{D}_{2}$ mesocortical pathway to the anterior cingulate cortex (ACC). Walter and colleagues [9] theorize that two consequences of diminished ACC activity are poor conscious emotional awareness and the inability to attribute bodily sensations of arousal to consciously perceived emotions; these consequences are, respectively, suggestive of the two most common features of alexithymia, inattention to emotion and poor clarity of emotion. Corroborating this model are neuroimaging data that implicate a compromise in ACC structure and function across facets of alexithymia [13-16].

The extant neurogenetic work is promising in light of the Walter et al. [9] theory, but more research is needed to explicate the relationships between BDNF- and DA-related genes in the alexithymia phenotype. To date, no one has examined the BDNF C270T polymorphism in the context of alexithymia or its related processes, although research is beginning to explore relationships between C270T and cognition in psychiatric and neurological populations [17-20]. The current study addresses this gap by examining the $\mathrm{C} 270 \mathrm{~T}$ polymorphism in relation to alexithymic dimensions and its potential interactions with three DA-related genes: the COMT and ANKK1 genes, as described earlier, and a gene coding for the dopamine transporter (DAT) that modulates DA availability. DAT, which is responsible for DA reuptake from extracellular space, is regulated by the DAT1 gene, located on chromosome $5 \mathrm{p} 15.3$. The DAT1 gene displays a polymorphic variable number of tandem repeats (VNTR) within the 3' untranslated region where 40 base pairs are repeated between three and thirteen times [21]. The 9- and 10-repeat genotypes are the most common in the general population [22], with the 10-repeat form associated with decreased DA transmission relative to the 9-repeat form [23]. As DAT is described as the most efficient way to clear the synapse of DA [24], examination of the DAT1 gene will help characterize any relationship between BDNF, DA, and alexithymic processes.

The multidimensionality of the alexithymia construct, however, complicates interpretation of genotype/phenotype data, as there is considerable overlap of alexithymia with the related constructs of emotional intelligence and mood awareness [25]. Factor analytic research has suggested that different facets of alexithymia have unique cognitive and emotion processing correlates $[25,26]$. Therefore, rather than measure alexithymia at the superordinate level, this study makes use of empirically-derived dimensions, re- sulting from principal components analysis of three closelyrelated self-report scales, to permit analysis of gene/alexithymia relationships at the facet level. As alexithymia is a dimensional personality trait, much in the same way as are extroversion and neuroticism, sampling from a normal population is appropriate for the present study. In order to control for the effects of confounding variables, we specifically chose a sample of community adults who were free of neurological and psychiatric conditions (e.g., traumatic brain injury, neurodegenerative disorders, posttraumatic stress disorder, addictions) for which alexithymia is among the known sequelae [27]. The reported association between the BDNF Val66Met polymorphism and alexithymia leads us to anticipate a similar relationship involving the BDNF C270T polymorphism. However, without knowing the intracellular mechanisms by which C270T exerts an influence on BDNF, it is difficult to predict a priori whether $\mathrm{C}$ or $\mathrm{T}$ allele carriers will have elevated scores and, if so, in which alexithymia-related domain(s). Given the previous research that implicates decreased dopaminergic function in alexithymia, we hypothesize interacting effects with the COMT, ANKK1, and DAT1 genes such that COMT Val, ANKK1 A1, and DAT1 10-repeat carriers, respectively, will exhibit greater emotion processing deficits.

\section{Method}

\subsection{Participants}

A community sample of 130 (79 women; 51 men) genetically-unrelated adults was recruited through newspaper advertisements. Ethnic diversity was consistent with local population norms: $87 \%$ White or Caucasian, 5\% Black or African-American, $6 \%$ Asian or Asian-American, and $2 \%$ Hispanic/Latino. Age ranged from 18 to $64 \mathrm{yr}$ $(\mathrm{M}=32.3, \mathrm{SD}=13.4)$, and educational achievement ranged from 11 to $26 \mathrm{yrs}(\mathrm{M}=15.8, \mathrm{SD}=2.6)$. The protocol was consistent with ethical guidelines of the Declaration of Helsinki and was approved by the local Institutional Review Board. Written informed consent was obtained per participant. Rule-out criteria for participation included English as a second language, color-blindness, presence of a diagnosed psychiatric illness and/or history of psychiatric treatment, history of significant neurological illness or brain injury, history of medical conditions with known cognitive or emotional sequelae, substance abuse history, and use of psychoactive substances within the last month. Psychiatric screening was done with the Mini International Neuropsychiatric Interview (M.I.N.I.) [28].

\subsection{Procedure}

Participants completed self-report questionnaires designed to assess alexithymia and its related constructs: the To- 
ronto Alexithymia Scale (TAS-20) [1], the Trait Meta Mood Scale (TMMS) [29], and the Mood Awareness Scale (MAS) [30]. DNA was obtained for some participants $(n=105)$ with peripheral blood samples, drawn via standard venipuncture by a certified phlebotomist, and with cheek buccal swabs for other participants $(n=25)$. All participants completed the protocol in individual sessions.

\subsection{Self-Report Measures}

The TAS-20 is a 20 -item scale that consists of three subscales: Difficulty Identifying Feelings (e.g., "When I am upset, I don't know if I am sad, frightened, or angry"), Difficulty Describing Feelings (e.g., "It is difficult for me to find the right words for my feelings"), and ExternallyOriented Thinking (e.g., "I find examinations of my feelings useful in solving personal problems"). Higher subscale scores reflect worse ability. Each subscale has shown adequate internal consistency, with alpha coefficients of $0.78,0.75$, and 0.66 , respectively [31]. The TAS-20 has also demonstrated good test-retest reliability in nonpatient samples across cultures [32].

The TMMS is a 30 -item scale that consists of three subscales: Attention to Feelings (e.g., "I don't think it's worth paying attention to your emotions or moods"), Clarity of Feelings (e.g., "Sometimes I can't tell what my feelings are"), and Mood Repair (e.g., "No matter how bad I feel, I try to think about pleasant things"). Lower subscale scores indicate worse ability. The TMMS has shown adequate internal consistency (full scale alpha coefficient $=0.82$ ), and internal reliabilities per subscale $(0.86,0.87$, and 0.82 , respectively) [29].

The MAS is a 10 -item scale of meta-emotion skills that consists of two subscales: Mood Monitoring (e.g., "I am sensitive to changes in my mood") and Mood Labeling (e.g., "Right now I know what kind of mood I'm in"). Lower subscale scores reflect worse ability. Both subscales have respectively shown adequate internal consistency $(0.88$ and 0.77$)$ and test-retest reliability $(0.94$ and 0.76) [33].

\subsection{Genoptying}

DNA extraction was completed with the Gentra Puregene Blood Kit (Qiagen: Valencia, California, USA) or with the QIAmp DNA Mini Kit (Qiagen), as appropriate. Genotyping for BDNF C270T, COMT (rs4680), and ANKK1 (rs1800497) was done at Dartmouth-Hitchcock Medical Center in Lebanon, New Hampshire, USA with TaqMan assays using pre-designed, allele-specific primer and probes from Applied Biosystems (Foster City, California, USA). All primers and probes were used at final concentrations of $900 \mathrm{nM}$ and $200 \mathrm{nM}$, respectively, in 1× TaqMan Universal PCR Master Mix (Applied Biosystems) on a
7500 Fast Real-Time PCR System using allelic discrimination analysis with the 7500 Software v2.0 (Applied Biosystems).

Genotyping for DAT1 was completed at Bates College in Lewiston, Maine, USA. The polymerase chain reaction (PCR) was run on an Eppendorf Mastercycler Pro thermal cycler (Hauppauge, New York, USA) using the following primers: DATVNTRF: 5'-TGT GGT GTA GGG AAC GGC CTG AGA-3' and DATVNTRR: 5'-CTT CCT GGA GGT CAC GGC TCA AGG-3'. Reactions were carried out in volumes of $50 \mu \mathrm{L}$ containing $22 \mu \mathrm{L}$ of sterile $\mathrm{H}_{2} \mathrm{O}, 8 \mu \mathrm{L}$ of $4 \mathrm{mM} \mathrm{MgCl} \mathrm{M}_{2}$ (New England BioLabs: Ipswitch, Massachusetts, USA), $5 \mu \mathrm{L}$ of 10X Taq Buffer (New England BioLabs), $5 \mu \mathrm{L}$ of dNTP mixture containing $10 \mathrm{mM}$ of each nucleotide (New England BioLabs), $2 \mu \mathrm{L}$ of each $0.5 \mu \mathrm{M}$ primer (Integrated DNA Technologies: Coralville, Iowa, USA), $5 \mu \mathrm{L}$ of DNA solution (QIAmp Qiagen kit), and $1 \mu \mathrm{L}$ of $5 \mathrm{U} / \mu \mathrm{L}$ Taq DNA polymerase (New England BioLabs). Each reaction underwent an initial denaturation at $94^{\circ} \mathrm{C}$ for 5 minutes, followed by 30 cycles of denaturation at $94^{\circ} \mathrm{C}$ for 30 seconds, annealing at $59^{\circ} \mathrm{C}$ for 30 seconds, and extension at $72^{\circ} \mathrm{C}$ for 45 seconds, with a final extension at $72^{\circ} \mathrm{C}$ for 10 minutes. Gel analysis used $2 \%$ low-melt agarose (BioRad: Hercules, California, USA) containing ethidium bromide. Genotypes were determined by comparison to a standard 100 base-pair ladder (New England BioLabs).

\section{Results}

\subsection{Genotyping Distributions}

Since not all participants provided consent for genetic assay of all four polymorphisms, total sample sizes vary slightly. BDNF C270T genotyping indicated a sample composition of 63 individuals with the $\mathrm{C} / \mathrm{C}, 43$ with the $\mathrm{C} / \mathrm{T}$, and 2 with the T/T genotype. To conserve statistical power, only individuals with the $\mathrm{C} / \mathrm{C}$ and $\mathrm{C} / \mathrm{T}$ genotypes were included for further analysis. COMT genotyping identified $30 \mathrm{Val} / \mathrm{Val}$, $52 \mathrm{Val} / \mathrm{Met}$, and $27 \mathrm{Met} / \mathrm{Met}$ individuals. Consistent with other researchers [10] and in order to maximize cell sizes, we condensed participants into Val homozygous and Met carrier (i.e., Met/Met and Val/Met) groups. DAT1 gene assay revealed 62 participants with the $10 / 10,55$ with the $9 / 10,6$ with the $9 / 9,4$ with the $10 / 11,1$ with the $3 / 3,1$ with the $8 / 10$, and 1 with the $9 / 11$ genotype. Like Garcia-Garcia and colleagues [34], we retained the 10/10 group and combined the $9 / 9$ and $9 / 10$ groups. Finally, ANKK1 testing indicated 5 individuals with the A/A, 35 with the $A / G$, and 66 with the G/G genotype; the $A / A$ and $A / G$ groups were subsequently combined. Distributions of each genotype was consistent with the Hardy-Weinberg equilibrium (all $p=\mathrm{ns}$ ), and all allelic groups were comparable in terms of age, gender, and 
years of education (all $p=\mathrm{ns}$ ).

\subsection{Data Reduction of Alexithymia Variables}

Principal component analysis (PCA) was performed to identify the latent factors among the TAS-20, TMMS, and MAS subscales. The questionnaire data met the standards of the Kaiser-Meyer-Olkin measure of sampling adequacy $(0.82)$ and Bartlett's test of sphericity (approximate $\left.\chi^{2}=575.4, \mathrm{df}=28, p<0.001\right)$ recommended for PCA [35]. Varimax rotation was used, and the number of factors retained was determined by the Kaiser criterion and visual inspection of the Scree plot. Two factors emerged, which together accounted for $70.1 \%$ of the total variance. Based on the pattern of factor loadings (Table 1), the first and second components were renamed Clarity of Emotion and Attention to Emotion, respectively. Given a modest sample size in relation to the number of subscale variables entered into PCA, we employed a rigorous cut-off criterion of $|0.8|$ for factor loading interpretation as recommended by Stevens [36] and Guadagnoli and Velicer [37]. The factor score coefficients were estimated using the Anderson-Rubin method [38], a modification of the Bartlett method that ensures orthogonality of the estimated factors. Like $z$-scores, these factor score coefficients have a mean of 0 and SD of 1 such that positive and negative values of the Clarity of Emotion and Attention to Emotion variables reflect above and below average skills, respectively.

\subsection{Simple Correlations}

Point-biserial correlations (two-tailed) were performed to provide an initial assessment of the relationships between genotypes, the extracted PCA factors, and demographic variables of age, sex, and level of education. The BDNF C270T polymorphism was related to Clarity of Emotion, $r=0.26, p=0.008$, but not to Attention to Emotion, $r=$ $0.04, p=$ ns. The COMT, DAT1, and ANKK1 polymorphisms showed no relationship with either emotion variable. There was no association among the genotypes groups and any of the demographic variables.

\subsection{BDNF C270T Effects}

A multivariate analysis of variance (MANOVA) with C270T genotype as a grouping factor was conducted for the extracted PCA factors. The full model was significant, Wilks' $\lambda=0.93, F(2,101)=3.80, p=0.03$, partial $\eta^{2}=$ 0.07 . Tests of between-subjects effects revealed that the $\mathrm{C} / \mathrm{C}$ group had lower Clarity of Emotion scores relative to the $\mathrm{C} / \mathrm{T}$ group, $F(1,102)=7.27, p=0.008$, partial $\eta^{2}$ $=0.07$ (Table 2). Attention to Emotion score was comparable across groups. The full model remained significant after covarying for COMT, DAT1, and ANKK1, Wilks' $\lambda=0.89, F(2,86)=5.32, p=0.007$, partial $\eta^{2}=0.11$,
Table 1. Factor loadings for alexithymia-related measures.

\begin{tabular}{lcc}
\hline & \multicolumn{2}{c}{ Rotated Components } \\
\hline Variable & $\mathrm{CE}$ & $\mathrm{AE}$ \\
\hline Eigenvalue & 4.0 & 1.6 \\
Variance explained & $49.6 \%$ & $20.5 \%$ \\
TAS-20 Difficulty Identifying Feelings & -0.88 & 0.01 \\
TAS-20 Difficulty Describing Feelings & -0.76 & -0.39 \\
TAS-20 Externally-Oriented Thinking & -0.20 & -0.85 \\
TMMS Attention to Feelings & 0.18 & 0.82 \\
TMMS Clarity of Feelings & 0.86 & 0.20 \\
TMMS Mood Repair & 0.51 & -0.01 \\
MAS Mood Monitoring & 0.02 & 0.87 \\
MAS Mood Labeling & 0.81 & 0.41 \\
\hline
\end{tabular}

Note. Component loadings $\geq|0.8|$ were considered significant. CE $=$ Clarity of Emotion; $\mathrm{AE}=$ Attention to Emotion; TAS-20 = Toronto Alexithymia Scale; TMMS $=$ Trait Meta-Mood Scale MAS $=$ Mood Awareness Scale.

Table 2. Subscales across BDNF C207T genotype groups.

\begin{tabular}{lcccc}
\hline & \multicolumn{2}{c}{ C/C group } & \multicolumn{2}{c}{ C/T group } \\
\hline Variable & M & SD & M & SD \\
\hline TAS-20 Difficulty Identifying Feelings & 13.8 & 5.4 & 11.6 & 3.6 \\
TAS-20 Difficulty Describing Feelings & 12.6 & 4.6 & 11.0 & 4.1 \\
TAS-20 Externally-Oriented Thinking & 17.7 & 4.9 & 17.3 & 4.7 \\
TMMS Attention to Feelings & 51.9 & 7.5 & 52.9 & 8.8 \\
TMMS Clarity of Feelings & 40.0 & 7.4 & 44.0 & 6.5 \\
TMMS Mood Repair & 22.7 & 4.6 & 23.9 & 4.7 \\
MAS Mood Monitoring & 18.2 & 6.3 & 19.5 & 6.2 \\
MAS Mood Labeling & 22.3 & 4.3 & 24.5 & 4.2 \\
Clarity of Emotion latent factor score & -0.2 & 1.1 & 0.3 & 0.8 \\
Attention to Emotion latent factor score & -0.1 & 1.0 & 0.0 & 1.1 \\
\hline
\end{tabular}

Note. TAS-20 $=$ Toronto Alexithymia Scale; TMMS $=$ Trait Meta-Mood Scale; MAS $=$ Mood Awareness Scale.

with the $\mathrm{C} / \mathrm{C}$ group again showing lower Clarity of Emotion scores relative to the $\mathrm{C} / \mathrm{T}$ group, $F(1,87)=8.80, p$ $=0.004$, partial $\eta^{2}=0.09$.

\subsection{Gene-Gene Interaction Effects}

To assess hypothesized genetic interactions, separate twoway analyses of variance (ANOVA) were performed with $\mathrm{C} 270 \mathrm{~T}$ genotype as one grouping factor and the COMT genotype (Val/Val versus Met carrier groups), DAT1 geno- 
type (10/10 versus a combined $9 / 9$ and $9 / 10$ group), and ANKK1 genotype (G/G versus A carrier groups), respectively, as the other. Attention to Emotion was dropped as a dependent variable given its negligible relationship with C270T. Clarity of Emotion was retained as the single dependent variable of interest. Similar to other exploratory behavioral genetics studies $[10,39]$ and given that a relationship between $\mathrm{C} 270 \mathrm{~T}$ and alexithymia has not previously been established in the literature, we chose not to apply a Bonferroni correction for multiple comparisons $(p<0.05 / 3=0.017)$ in these analyses; adjusting the alpha threshold for significance might inappropriately increase the possibility that a real effect is missed among these data [40]. In all three analyses, a main effect emerged of C270T polymorphism on Clarity of Emotion, but there was no interaction between C270T and any of the COMT, DAT1, or ANKK1 genotypes ${ }^{1}$.

\section{Discussion}

This study is the first known exploratory investigation of the BDNF C270T polymorphism in the context of alexithymia. In consideration of a theory [9] that postulates dopaminergic activity as an important mediating step between BDNF and alexithymia, we included three DA-related genes in our analysis: the COMT, DAT1, and ANKK1 genes. Mindful of the multidimensional nature of alexithymia and its substantial overlap with the concepts of emotional intelligence and mood awareness, we used PCA to identify latent factors common to all three constructs. These principal components, Clarity of Emotion (i.e., the degree to which one understands and can label emotional states) and Attention to Emotion (i.e., the degree to which one attends to emotions), demonstrated different relationships with the BDNF C270T polymorphism, which is consistent with other research that highlights unique sets of external correlates for these affective dimensions $[25,26]$.

In the present sample, the $\mathrm{C} 270 \mathrm{~T} \mathrm{C/C}$ genotype group exhibited lower Clarity of Emotion scores relative to the $\mathrm{C} / \mathrm{T}$ genotype group, and this effect held after covarying for COMT, DAT1, and ANKK1 genotypes, a finding that was not confounded by age, gender, or education differences. The three DA-related genes themselves did not relate to either alexithymia facet, nor did these genes interact with the C270T polymorphism to predict Clarity of Emotion scores. As the C270T polymorphism explains up to $11 \%$ of the variance in Clarity of Emotion, there clearly are other variables that account for the rest. These other variables, however, do not appear to include the COMT, DAT1, and ANKK1 genes. It is also unlikely

\footnotetext{
${ }^{1}$ Although the majority of participants are of European ancestry, there are multiple ethnic groups represented in the rest of the sample. Given the known effects of population stratification in genetics, we repeated this analysis with just the homogenous European ethnic group $(n=90)$. The same patterns emerged.
}

that psychiatric or neurological variables contribute differentially to this effect, as only healthy adults, screened for conditions that are often comorbid with alexithymia, were included in this study.

Despite a modest, multiethnic sample, the present study suggests a role for BDNF in modulating aspects of alexithymia, particularly the tendency to have poor insight into one's emotional states. The relationship between the C270T polymorphism and Clarity of Emotion is broadly consistent with the recent finding by Walter et al. [9] of a connection between the BDNF Val66Met polymorphism and the TAS-20 subscale Difficulty Identifying Feelings. Taken together, this is evidence of an association between two different BDNF polymorphisms and nearly identical phenotypic traits. Although $\mathrm{C} 270 \mathrm{~T}$ appears to be functional, we do not yet know the molecular mechanisms by which it exerts an effect. Like Met substitution for Val66Met (for review, see [41]), the $\mathrm{C} / \mathrm{C}$ genotype might lead to trafficking defects in BDNF secretory pathways and, subsequently, reduce BDNF availability in the synapse. Future research with transgenic animal models is necessary to characterize the intra- and intercellular consequences of the genotypes. It is also important that future research on the BDNF gene should simultaneously assay the C270T and Val66Met polymorphisms to determine if they are being inherited as a haplotype.

When assessing a phenotypic change in tandem with a genetic variant, it is difficult to determine whether the genotype in question has a direct effect on the phenotype or whether the effect is mediated through a downstream functional change or interaction with another gene [41]. Walter and colleagues [9] address this possibility when they advance the idea that ACC activity is a proximal contributor to emotional clarity, which itself is governed in part by dopaminergic activity in the $\mathrm{D}_{2}$ mesocortical pathway, which, in turn, is influenced by varying BDNF levels. In this model, the BDNF genotype is a distal agent in a multi-step biological pathway. Contrary to our hypothesis and contrary to this model, we did not find an interaction between $\mathrm{C} 270 \mathrm{~T}$ and any of the DA-related genes. Although this is a major point of difference between the two studies, the present findings do not necessarily negate the Walter et al. [9] theory. Three possibilities exist to reconcile the discrepant results. First, it is possible that the C270T polymorphism, in its action on BDNF and independently of state-like changes in dopaminergic activeity, leads to structural changes beyond the anterior cingulate (e.g., PFC, medial temporal lobe, caudate nucleus) that are associated with alexithymia. Second, it is possible that some change in the DA system is still an important intermediary but that the $\mathrm{C} 270 \mathrm{~T}$ polymorphism interacts with genes outside of COMT, DAT, or ANKK1 (e.g., DRD1, DRD3, and DRD4). Third, it is possible that there is a non-DA neurotransmitter intermediary (e.g., 
serotonin, acetylcholine) that links the C270T polymerphism to clarity of emotion.

This list is by no means exhaustive, but it does highlight the neuropharmacological complexity of BDNF that makes interpretation of relationships between genetic variants and facets of emotion processing so challenging. Future research is needed not only to characterize the molecular basis of altered BDNF function by C270T but also to map structural and functional brain differences between $\mathrm{C} / \mathrm{C}$ and $\mathrm{C} / \mathrm{T}$ groups. Description of where and under what conditions these groups differ at the level of the brain will be an important step to refine theories of how BDNF influences alexithymia and why the C270T polymorphism shapes the clarity of emotion facet alone.

\section{Acknowledgements}

This work was supported by grants from Bates College and the Maine Institute of Human Genetics and Health (to N.S.K.) and Sigma Xi Scientific Research Society (to L.H.C.). The authors would like to thank Mary E. Hughes of Bates College in Lewiston, ME USA for phlebotomy and Gregory Tsongalis, Ph.D., Mary C. Schwab, Ph.D., Joel A. Lefferts, Ph.D., and Claudine L. Lefferts, Ph.D. of Dartmouth-Hitchcock Medical Center in Lebanon, New Hampshire, USA for genotyping consultation.

\section{REFERENCES}

[1] G. B. Taylor, M. Bagby and J. D. Parker, "Disorders of Affect Regulation: Alexithymia in medical and Psychiatric Illness," Cambridge University Press, New York City, 1997. doi:10.1017/CBO9780511526831

[2] J. L. King and B. Mallinckrodt, "Family Environment and Alexithymia in Clients and Non-Clients," Psychotherapy Research, Vol. 10, No. 1, 2000, pp. 78-86. doi:10.1080/713663595

[3] S. Koponen, T. Taiminen, K. Honkalampi, M. Joukamaa, H. Vinnamaki, T. Kurki, et al., "Alexithymia after Traumatic Brain Injury: Its Relation to Magnetic Resonance Imaging," Psychosomatic Medicine, Vol. 67, No. 5, 2005, pp. 807-812. doi:10.1097/01.psy.0000181278.92249.e5

[4] S. B. Zeitlin, R. J. McNally and K. L. Cassiday, "Alexithymia in Victims of Sexual Assault: An Effect of Repeated Traumatization?" American Journal of Psychiatry, Vol. 150, No. 4, 1993, pp. 661-663.

[5] J. K. Salminen, S. Saarijärvi, E. Äärelä, T. Toikka and J. Kauhanen, "Prevalence of Alexithymia and Its Association with Sociodemographic Variables in the General Population of Finland," Journal of Psychosomatic Research, Vol. 46, No. 1, 1998, pp. 75-82. doi:10.1016/S0022-3999(98)00053-1

[6] M. M. Jørgensen, R. Zachariae, A. Skytthe and K. Kyvik, "Genetic and Environmental Factors in Alexithymia: A Population-Based Study of 8,785 Danish Twin Pairs," Psychotherapy and Psychosomatics, Vol. 76, No. 6, 2007, pp. 369- 375. doi:10.1159/000107565
[7] E. M. Valera and H. Berenbaum, "A Twin Study of Alexithymia," Psychotherapy and Psychosomatics, Vol. 70, No. 5, 2001, pp. 239-246. doi:10.1159/000056261

[8] H. M. Abdolmaleky, C. L. Smith, J. R. Zhou and S. Thiagalingam, "Epigenetic Alterations of the Dopaminergic System in Major Psychiatric Disorders," Methods in Molecular Biology, Vol. 448, 2008, pp. 187-212. doi:10.1007/978-1-59745-205-2 9

[9] N. T. Walter, C. Montag, S. A. Markett and M. Reuter, "Interaction Effect of Functional Variants of the BDNF and DRD2/ANKK1 Gene Is Associated with Alexithymia in Healthy Human Subjects," Psychosomatic Medicine, Vol. 73, No. 1, 2011, pp. 23-28. doi:10.1097/PSY.0b013e31820037c1

[10] B. J. Ham, M. S. Lee, Y. M. Lee, M. K. Kim, M. J. Choi, K. S. Oh, et al., "Association between the Catechol$O$-Methyltransferase Val108/158Met Polymorphism and Alexithymia," Neuropsychobiology, Vol. 52, No. 3, 2005, pp. 151-154. doi:10.1159/000087846

[11] S. Hermes, J. Hennig, M. Stingl, F. Leichsenring and F. Leweke, "No Association between Catechol-O-MethylTransferase val158met Polymorphism and Alexithymia," Zeitschrift fur Psychosomatische Medizin und Psychotherapie, Vol. 57, No. 1, 2011, pp. 51-61.

[12] T. Pohjalainen, J. O. Rinne, K. Någren, P. Lehikoinen, K. Anttila, E. K. Syvälathi, et al., "The A1 Allele of the Human $\mathrm{D}_{2}$ Dopamine Receptor Gene Predicts Low $\mathrm{D}_{2}$ Receptor Availability in Healthy Volunteers," Molecular Psychiatry, Vol. 3, No. 3, 1998, pp. 256-260. doi:10.1038/sj.mp.4000350

[13] H. Gündel, A. Lopez-Sala, A. O. Ceballos-Baumann, J. Deus, N. Cardoner, B. Marten-Mittag, C. Soriano-Mas, et al., "Alexithymia Correlates with Size of the Right Anterior Cingulate," Psychosomatic Medicine, Vol. 66, No. 1, 2004, pp. 132-140. doi:10.1097/01.PSY.0000097348.45087.96

[14] H. Karlsson, P. Näätänen and H. Stenman, "Cortical Activation in Alexithymia as a Response to Emotional Stimuli," British Journal of Psychiatry, Vol. 192, 2008, pp. 32-38. doi:10.1192/bjp.bp.106.034728

[15] N. S. Koven, R. M. Roth, M. A. Garlinghouse, L. A. Flashman and A. J. Saykin, "Regional Gray Matter Correlates of Perceived Emotional Intelligence," Social, Cognitive, and Affective Neuroscience, Vol. 6, No. 5, 2010, pp. 582-590. doi: $10.1093 / \mathrm{scan} / \mathrm{nsq} 084$

[16] C. Montag, B. Weber, E. Jentgens, C. Elger and M. Reuter, "An Epistasis Effect of Functional Variants on the BDNF and DRD2 Genes Modulates Grey Matter Volume of the Anterior Cingulate Cortex in Healthy Humans," Neuropsychologia, Vol. 48, No. 4, 2010, pp. 1016-1021. doi:10.1016/j.neuropsychologia.2009.11.027

[17] H. Kunugi, A. Ueki, M. Otsuka, K. Isse, H. Hirasawa and K. Kato, "A Novel Polymorphism of the Brain-Derived Neurotrophic Factor (BDNF) Gene Associated with LateOnset Alzheimer's Disease," Molecular Psychiatry, Vol. 6, No. 1, 2001, pp. 83-86. doi:10.1038/sj.mp.4000792

[18] A. Parsian, R. Sinha, B. Racette, J. H. Zhao and J. S. Perlmutter, "Association of a Variation in the Promoter Region of the Brain-Derived Neurotrophic Factor Gene 
with Familiar Parkinson's Disease," Parkinsonism and Related Disorders, Vol. 10, No. 4, 2004, pp. 213-219. doi:10.1016/j.parkreldis.2003.12.003

[19] A. Szczepankiewicz, M. Skibińska, J. Hauser, A. Leszczyńska-Rodziewicz, M. Dmitrzak-Weglarz, P. M. Czerski, et al., "Association Study of the Brain-Derived Neurotrophic Factor (BDNF) Gene C-270T Polymorphism with Bipolar Affective Disorder," Archives of Psychiatry and Psychotherapy, Vol. 8, 2006, pp. 31-39.

[20] G. Szekeres, A. Juhász, A. Rimanóczy, S. Kéri and Z. Janka, "The C270T Polymorphism of the Brain-Derived Neurotrophic Factor Gene Is Associated with Schizophrenia," Schizophrenia Research, Vol. 65, No. 1, 2003, pp. 15-18. doi:10.1016/S0920-9964(02)00505-4

[21] M. Nakatome, K. Honda, Z. Tun, Y. Kato, S. Harihara, K. Omoto, et al., "Genetic Polymorphism of the 3' VNTR Region of the Human Dopaminergic Function Gene DAT1 (Human Dopamine Transporter Gene) in the Mongolian Population," Human Biology, Vol. 68, No. 4, 1996, pp. 509-515.

[22] A. M. Kang, M. A. Palmatier and K. K. Kidd, "Global Variation of a 40-bp VNTR in the 3' Untranslated Region of the Dopamine Transporter Gene (SLC6A3)," Biological Psychiatry, Vol. 46, No. 2, 1999, pp. 151-160. doi:10.1016/S0006-3223(99)00101-8

[23] J. Mill, P. Asherson, C. Browes, U. D’Souza and I. Craig, "Expression of the Dopamine Transporter Gene Is Regulated by the 3' UTR VNTR: Evidence from Brain and Lymphocytes Using Quantitative RT-PCR," American Journal of Medical Genetics, Vol. 114, No. 8, 2002, pp. 975-979. doi:10.1002/ajmg.b.10948

[24] A. Diamond, "Consequences of Variations in Genes that Affect Dopamine in Prefrontal Cortex," Cerebral Cortex, Vol. 17, No. 1, 2007, pp. 161-170. doi:10.1093/cercor/bhm082

[25] E. Coffey, H. Berenbaum and J. G. Kerns, "The Dimensions of Emotional Intelligence, Alexithymia, and Mood Awareness: Associations with Personality and Performance on an Emotional Stroop Task," Cognition and Emotion, Vol. 17, No. 4, 2003, pp. 671-679. doi:10.1080/02699930302304

[26] N. S. Koven and W. Thomas, "Mapping Facets of Alexithymia to Executive Dysfunction in Daily Life," Personality and Individual Differences, Vol. 49, No. 1, 2010, pp. 24-28. doi:10.1016/j.paid.2010.02.034

[27] R. Becerra, A. Amos and S. Jongenelis, "Organic Alexithymia: A Study of Acquired Emotional Blindness," Brain Injury, Vol. 16, No. 7, 2002, pp. 633-645. doi:10.1080/02699050110119817

[28] D. V. Sheehan, Y. Lecrubier, K. Harnett-Sheehan, P. Amorim, J. Janavs, E. Weiller, et al., "The Mini International Neuropsychiatric Interview (M.I.N.I.): The Development and Validation of a Structured Diagnostic Psychiatric Interview for DSM-IV and ICD-10," Journal of Clinical Psychiatry, Vol. 59, No. 20, 1998, pp. 22-23.

[29] P. Salovey, J. D. Mayer, S. L. Goldman, C. Turvey and T. P. Palfai, "Emotional Attention, Clarity, and Repair: Ex- ploring Emotional Intelligence Using the Trait metaMood Scale," In: J. W. Pennebaker, Ed., Emotion, Disclosure, and Health, American Psychological Association, Washington DC, 1995, pp. 125-154.

doi:10.1037/10182-006

[30] A. Swinkles and T. A. Giuliano, "The Measurement and Conceptualization of Mood Awareness: Monitoring and Labeling One's Mood States," Personality and Social Psychology Bulletin, Vol. 21, No. 9, 1995, pp. 934-950.

[31] R. M. Bagby, G. J. Taylor and J. D. A. Parker, "The Twenty-Item Toronto Alexithymia Scale: II. Convergent, Discriminant, and Concurrent Validity," Journal of Psychosomatic Research, Vol. 38, No. 1, 1994, pp. 33-40. doi:10.1016/0022-3999(94)90006-X

[32] C. G. Kooiman, P. Spinhoven and R. W. Trijsburg, "The Assessment of Alexithymia: A Critical Review of the Literature and a Psychometric Study of the Toronto Alexithymia Scale-20," Journal of Psychosomatic Research, Vol. 53, No. 6, 2002, pp. 1083-1090. doi:10.1016/S0022-3999(02)00348-3

[33] M. B. Harris, "Correlates and Characteristics of Boredom Proneness and Boredom," Journal of Applied Social Psychology, Vol. 3, No. 3, 2000, pp. 576-598. doi:10.1111/j.1559-1816.2000.tb02497.x

[34] M. Garcia-Garcia, F. Barceló, I. C. Clemente and C. Escera, "The Role of the Dopamine Transporter DAT1 Genotype on the Neural Correlates of Cognitive Flexibility," European Journal of Neuroscience, Vol. 31, No. 4, 2010, pp. 754-760. doi:10.1111/j.1460-9568.2010.07102.x

[35] B. G. Tabachnick and L. S. Fidell, "Using Multivariate Statistics" 5th Edition, Pearson Education, Boston, 2007.

[36] J. P. Stevens, "Applied Multivariate Statistics for the Social Sciences," 4th Edition, Lawrence Erlbaum Associates, Mahwah, 2002.

[37] E. Guadagnoli and W. F. Velicer, "Relation of Sample Size to the Stability of Component Patterns," Psychological Bulletin, Vol. 103, No. 2, 1988, pp. 265-275. doi:10.1037/0033-2909.103.2.265

[38] R. D. Anderson and H. Rubin, "Statistical Inference in Factor Analysis," Proceedings of the 3rd Berkeley Symposium of Mathematical Statistics and Probability, Vol. 5, 1956, pp. 111-150.

[39] H. A. Wishart, R. M. Roth, A. J. Saykin, H. Rhodes, G. J. Tsongalis, K. A. Pattin, et al., "COMT Val158Met Genotype and Individual Differences in Executive Function in Healthy Adults," Journal of the International Neuropsychological Society, Vol. 17, No. 1, 2011, pp. 174-180. doi: $10.1017 / \mathrm{S} 1355617710001402$

[40] K. J. Rothman, "No Adjustments Are Needed for Multiple Comparisons," Epidemiology, Vol. 1, No. 1, 1990, pp. 43-46. doi:10.1097/00001648-199001000-00010

[41] K. G. Bath and F. S. Lee, "Variant BDNF (Val66Met) Impact on Brain Structure and Function," Cognitive, Affective, and Behavioral Neuroscience, Vol. 6, No. 1, 2006, pp. 79-85. doi:10.3758/CABN.6.1.79 